\title{
RECENT RESULTS FROM AND FUTURE PLANS FOR THE VISA II SASE FEL
}

\author{
G. Andonian, R. Agustsson, P. Frigola, A. Murokh, C. Pellegrini, \\ S.Reiche, J. Rosenzweig, G. Travish, UCLA, Los Angeles, CA 90095, USA \\ M. Babzien, I. Ben-Zvi, V. Litvinenko, V. Yakimenko, BNL, Upton, NY 11973, USA \\ I. Boscolo, S. Cialdi, A. Flacco, INFN-Milano, Milano, Italy \\ M. Ferrario, L. Palumbo, C. Vicario, INFN/LNF, Frascati, Roma, Italy \\ J. Huang, PAL, Pohang, S. Korea
}

\section{Abstract}

The VISA II (Visible to Infrared SASE Amplifier) project, a consequent experiment to the succesful VISA enterprise, entails the use of a chirped electron beam to drive a high gain SASE FEL. The resulting ultra-short pulses will be characterized using an advanced FROG (Frequency Resolved Optical Gating) technique, as well as a double differential spectrum (angle/wavelength) diagnostic. Implementation of sextupole corrections to the longitudinal aberrations affecting the high energy-spread chirped beam during transport to the VISA undulator is studied. Start-end simulations, including radiation diagnostics, are discussed. Initial experimental results involving a highly chirped beam transported without sextupole corrections, the resulting high gain lasing, and computational analysis are briefly reported.

\section{INTRODUCTION}

As the promise of X-ray free electron lasers (FEL) comes close to realization $[1,2]$, the creation and diagnosis of ultra-short pulses is of great relevance to the SASE (Self-Amplified Spontaneous Emission) FEL (SelfAmplified Spontaneous Emission) community. Femtosecond long, Ångstrom wavelength radiation will provide the spatial resolution desired for scientific investigations at the time scales of atomic process, such as biological sampling (single molecule diffraction) [3]. Such sources are designed to operate at hundred femtosecond pulse lengths with a clear demand to push to shorter time scales.

A proposal to obtain such ultra-short pulses [4], by manipulating frequency chirped FEL output, serves as the afflatus for the VISA II experiment. The aim of the VISA II project is to operate the SASE FEL with the highest electron beam chirp allowable at the upgraded systems of the Brookhaven National Laboratory Accelerator Test Facility (BNL ATF). The production, and subsequent measurement, of strongly chirped SASE FEL radiation based on electron beam chirping is the objective of this multi-institutional collaborative effort.

\section{EXPERIMENT DESCRIPTION}

\section{Summary of Prior Results}

The VISA experiment layout is described in detail in Ref. [5]. The VISA project succesfully demonstrated saturation of a SASE FEL within a 4 meter undulator at 840 $\mathrm{nm}$. An electron bunch compression mechanism, exploiting the nonlinear properties of transport along the dispersive line of the ATF, was discovered to increase the peak current from 55 A to $240 \mathrm{~A}$. Electron beam dynamics in the gun, transport, and undulator were modeled with the simulation codes PARMELA [6], ELEGANT [7], and GENESIS 1.3 [8] respectively. SASE FEL properties, such as pulse energy, profile, and angular distribution were modeled with GENESIS. The harvest of the start-end suite of codes was accurately benchmarked against experimental findings. The complete characterization of the SASE FEL properties included gain lengths, spectra, energies and observation of nonlinear harmonics [9].

\section{Present Results and Analysis}

Follow-up measurements at VISA took place with the employ of a highly chirped electron pulse, with longitudinal compression, and subsequent FEL amplification. An anomolously large bandwidth, up to $15 \%$ full width, was observed at high gain accompanied by atypical far-field angular radiation patterns (Fig. 1).

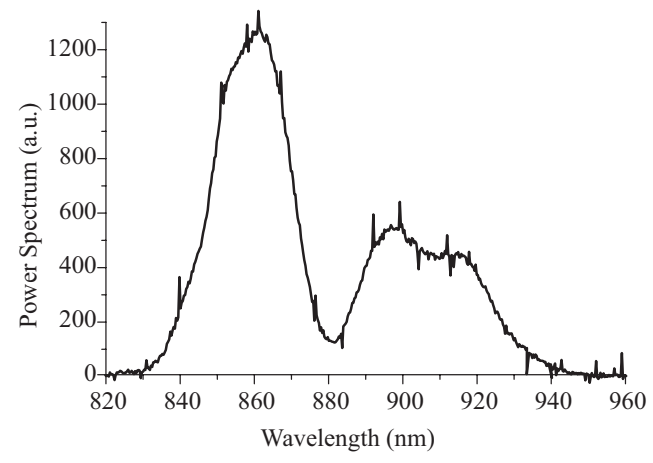

Figure 1: Sample shot of observed SASE FEL spectrum with anomalously large bandwidth. Start-end simulations were able to reproduce key facets of the spectrum. 
An electron bunch, of approximately $330 \mathrm{pC}$, with $1.7 \%$ energy spread, was propogated through the undulator. Due to nonlinear compression, the peak current of the pulse reached $300 \mathrm{~A}$. The compression, as well as the SASE FEL output, was extremely stable and insensitive to RF and laser timing jitter. After transport, the electron beam displayed a highly nonlinear longitudinal phase space. The average measured SASE radiated energy was approximately $2 \mu \mathrm{J}$ and displayed an extraordinary wavelength distribution. The spectrum is notable for a characteristic double peak structure, accompanied by a mean bandwidth value of $12 \%$ full width (in excess of $100 \mathrm{~nm}$ ), as seen in Fig. 1.

Start-end simulations were able to reproduce the unique features of the radiation and held the key for the physical understanding behind this previously unobserved FEL amplification. Indeed, GENESIS simulations reproduced the large bandwidth and the associated double spiked structure. The secondary spike was due to amplification of an off-axis mode. The mode was excited by the non-ideal centroid and envelope motion of the beam through the undulator's quadrupole focusing lattice. Mismatches of the beam's lasing core to the focusing lattice caused excursions in beam size near $30 \mu \mathrm{m}$ in both transverse planes. Additional transverse motion causes a red-shift in the radiated wavelength and an ensuant inclination to ampify the off-axis modes.

\section{Double Differential Spectrometer}

An additional diagnostic has been developed to unfold the relationship between frequency and angle of the FEL radiation. This diagnostic employs the use of an adjustible slit, two cylindrical lenses for focusing, a set of gratings, and a CCD. A slice of the FEL ouput is passed through the slits, then focused onto the gratings $\left(1200 \mathrm{in}^{-1}\right)$. The resulting image displays the photon beam with frequency along one axis, and transverse angle along the other axis. The double-differential spectrometer is calibrated with the spectral lines of an Argon lamp. The resultant outcome is a direct study of the intensity of the beam presented in a most familiar form, $\frac{d^{2} I}{d \omega d \theta}$, the double differential spectrum.

Some recent data of this diagnostic is shown in Fig. 2. The overall parabolic structure of the beam in $(\theta, \omega)$ space is evident, with even richer multi-mode patterns also present.
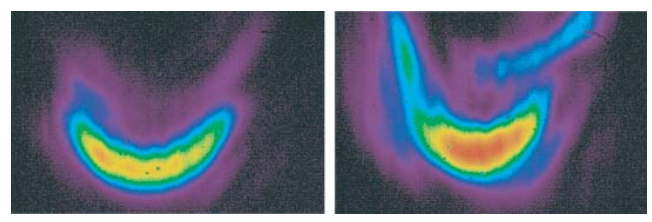

Figure 2: The overall parabolic structure (left) is clear in the images from the double differential spectrometer, where the angle is represented along the horizontal axis and the frequency along the vertical. More exotic and richer structures have also been observed (right).

Start-end simulations have been carried out to examine these results further. The GENESIS post-processing unit has been upgraded to compute the double differential spectrum for the experimental parameters at VISA. Preliminary runs display the aforementioned parabolic structure and even some higher order modes are apparent, confirming the dual lasing modes observed in previous runs (Fig. 3).

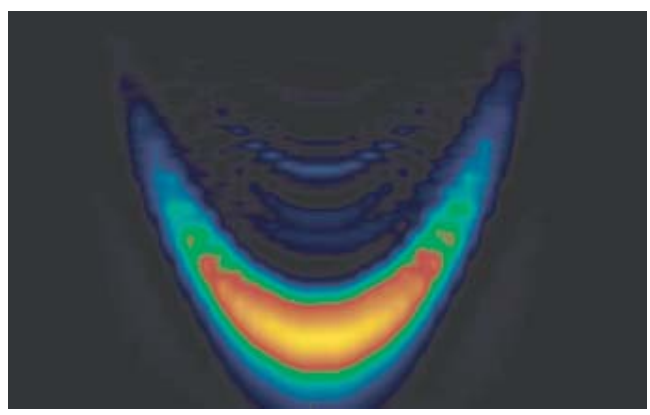

Figure 3: Output from a GENESIS simulation confirms the parabolic nature observed in VISA. Angles lie along the horizontal axis, frequencies along the vertical.

\section{Far-field Angular Distribution}

A unique ancillary component of the experiment is the observed angular distribution profile in the far-field. This measurement was made by allowing the output radiation, to propogate, without focusing lenses, to a screen approximately $3 \mathrm{~m}\left(10 \mathrm{Z}_{R}\right)$ away. The patterns observed were hollow in nature, like previous VISA results, but more pronunced in angle. Spiral shaped patterns accompanied the hollow modes. The helicity of this patterns will be studied via a mode converter, described later in this note.
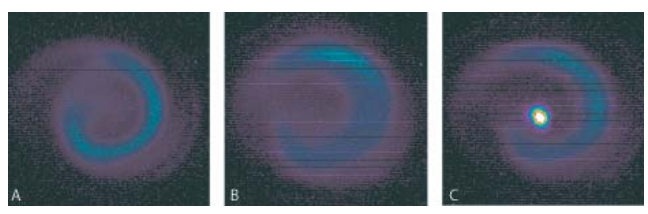

Figure 4: Far-field angular distribution profiles from VISA demonstrating the atypical spirality and helicity $(A, B)$ and superimposed with a red diode alignment laser for reference $(C)$.

\section{UPCOMING MEASUREMENTS}

\section{Chirped Beam Operation}

The main goal of the VISA II is experiment is to inject a linearly chirped electron beam into the undulator to produce frequency chirped SASE FEL radiation. Although the bunch compression mechanism facilitates highgain lasing, it restricts the management of beam properties through transport. Preservation of the electron beam chirp applied at the linac will be accomplished by the manipulation of nonlinear lonigtudinal compression by the use of 
sextupole magnets placed at points of high horizontal dispersion. The sextupoles will mitigate second order effects, particularly by diminshing the $T_{566}$ element, of the transport matrix, to a negligible value [10].

Measuring the $T_{566}$ term directly presents a challenge as compression is required for high gain lasing in VISA II. The actual value that can be quantified is the $T_{166}$ element. This value is measured by comparing the effect of incremental changes in the magnetic fields of all magneto-optic elements along the beamline to the change in position of the electron beam centroid at a point of non-zero dispersion. The correlation between the $T_{166}$ and $T_{566}$ terms is used to quantify the effects of the sextupoles.
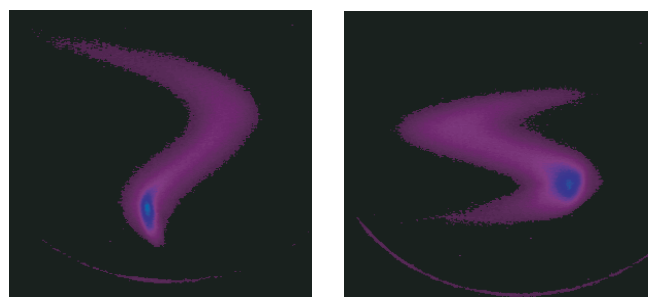

Figure 5: Two transverse profiles of the electron beam in the dispersive section of the transport with sextupole magnets energized. To create such extreme profiles the settings of the sextupoles were exaggerated from the ideal values computed using simulations.

\section{Optical Diagnostics}

Once electron beam chirp is preserved, subsequent SASE FEL lasing at high gain is achieved. The resulting radiation will be frequency chirped. A standard method of measuring the structure of short pulse, frequency chirped light is frequency resolved optical gating (FROG) [11]. For the expected parameters of the VISA II SASE FEL, FROG seems to be the most viable candidate.

One aspect of FROG, or GRENOUILLE (a close relative of FROG designed to simplifiy alignment issues by employing fewer, multi-purpose optical elements), that is a concern for the proposed measurement is the resolution of the spectrogram. For the radiated profile presented in previous results and properties noted in GENESIS simulations, namely the anomalously large spectral width and the hollow mode angular distribution pattern, the FROG technique needs modification to efficiently serve its purpose. The proposed modifications revolve around the GRENOUILLE diagnostic, which employs the use of a thick crystal with the dual purpose of recombination and dispersion of the split signal. The system is too constrained by the doubling crystal to properly resolve the radiation expected from the VISA II FEL. The thick lens must be replaced by a thin lens and a dedicated spectrometer. In addition, a larger (several megapixel) CCD must replace the camera that is used in the commercial version of this diagnostic.

Simulations concerning the feasibility of this measurement have been conducted. GENESIS outputs for the chirped beam case show a clear effect for idealized beam shapes. These results have been analyzed for varying degrees of chirp. Indeed, the inversion algorithm is robust enough to handle other exotic shapes and patterns which have been simulated and reconstructed via the commercial FROG software (Femtosoft) for the VISA II experiment.

\section{Mode Converter and Polarizer}

The investigation of hollow mode and spiral shaped farfield angular radiation patterns at VISA continues via a diagnostic mode converter. The mode converter is design to transform light wtih Hermite-Gaussian modes to LaguerreGaussian modes; the transfer of orbital angular momentum is the observable [12]. The $\pi / 2$ mode converter is constructed of two cyldrical lenses, separated by a distance of $d=\sqrt{2} f$, and the resultant light will have distinct observable properties.

The VISA project will also examine the study of Transistion Undulator Radiation (TUR) [13], the radiation emitted by the electron bunch as it passes through the entrance and exit of the undulator, due to its change in longitudinal velocity. In fact, this radiation is thought to be radially polarized, describing yet another possible explanation for the helicity of the observed far-field patterns from the planar undulator at VISA. The quantization of this effect requires minimal alteration of already existing diagnostics with the addition of grid polarizers to determine the polarization of the radiation.

This work supported by Dept. of Energy Contract no. DE-FG-98ER45693, and Office of Naval Research no. N000140210911

\section{REFERENCES}

[1] M. Cornacchia et al., Linac Coherent Light Source Design Study Report no. SLAC-521 (1998)

[2] TESLA-FEL, Deutsches Elektronen Synchrotron 2001-05 (2001)

[3] R. Neutze et al., Nature 406, 752 (2000)

[4] C. Schroeder et al., J. Opt. Soc. Am. B 19, 1782 (2002)

[5] A. Murokh et al., Phys. Rev. E 67, 066501 (2003)

[6] L.M. Young, J.H. Billen, Los Alamos National Laboratory Report No. LA-UR-96-1835 (rev. 2000)

[7] M. Borland, Advanced Photon Source LS-287 (2000)

[8] S. Reiche, Nucl. Instrum. Methods A 429, 243 (1999)

[9] A. Tremaine et al., Phys. Rev. Lett. 88, 204801 (2002)

[10] J. England et al., Phys. Rev. ST Accel. Beams 8, 012801 (2005)

[11] R. Trebino, Frequency Resolved Optical Gating, Kluwer Academic Publishers (2000)

[12] M.W. Beijersbergen et al., Optics Comm. 96, 123-132 (1993)

[13] S. Reiche et al., Procedings of the 2004 FEL Conference, 193-196, Trieste, Italy. 